\title{
Lipid Deposition in the Aorta of Adjuvant Arthritic Rats with Hypercholesterolemia
}

\author{
Masaru KUNITOMO, Yu YAMAGUCHI, Yoshiko FUTAGAWA, \\ Yoshiko HAMAGUCHI and Yoshio BANDÔ \\ Department of Pharmacology. Faculty of Pharmaceutical Sciences. \\ Mukogawa Women's University. Nishinomiya 663. Japan \\ Accepted July 7, 1986
}

\begin{abstract}
This study offers findings which should aid in the development of a convenient animal model of atherosclerosis. Inbred Fisher strain rats were fed an atherogenic diet containing $1.5 \%$ cholesterol and $0.5 \%$ chclic acid and given a single subcutaneous injection of adjuvant (Mycobacterium butyricum) into the base of the tail. The animals were maintained for 8 weeks. Rats given the atherogenic diet showed markedly increased serum cholesterol levels, and all of those given the adjuvant injection developed severe polyarthritis. Cholesterol feeding tended to delay the onset of arthritis and remarkably suppressed the inflammatory response, particularly in the early stage of development. This may have been due to the lowered lipid peroxide levels in the serum of rats fed the atherogenic diet. Adjuvant arthritis together with cholesterol feeding markedly increased the cholesterol content of the aorta, whereas either treatment alone had little effect. The amounts of the connective tissue components and minerals in the aorta were not changed by both treatments. These results show that early atherosclerosis could be produced under the conditions used and that chronic inflammation and hypercholesterolemia are principal factors in the pathogenesis.
\end{abstract}

Atherosclerotic lesions have been shown to develop as a response to injury to arterial endothelial cells with subsequent platelet adherence and vessel wall infiltration of platelet-derived growth factor and plasma lipoproteins (1). Potential sources of vascular injury include chemical factors such as chronic hyperlipidemia, immune complex, endotoxin, and mechanical factors (1-3). Inflammation of the arterial wall, particularly that resulting from immunologic injury, has been considered to be important in the development of atherosclerosis in man (4). The marked development of atherosclerosis in combination with collagen diseases has been observed in man $(5,6)$ and experimental animals (7).

Virág et al. (8) histologically established experimental atherosclerosis in rats using a combination of chronic inflammation, adjuvant arthritis, and cholesterol feeding. They also found that treatment with anti- inflammatory drugs moderates the induction of the atherosclerosis with considerable lipid deposition (9). Generally, rats are known to be resistant to the induction of atherosclerotic lesions. Therefore, the experimental atherosclerosis mentioned above is of great interest not only for studies on the cause and pathogenesis of the disease but also because rats can serve as a small and readily available animal model.

The purpose of the present study was to investigate the extent of lipid deposition in the aorta of rats treated with adjuvant and a high-cholesterol diet and to characterize the biochemical aspects in the serum and aorta of this model.

\section{Materials and Methods}

Animals and diet: Five-week-old male Fischer F344/N rats (Shizuoka Laboratory Animal Center) were housed in an airconditioned room $\left(23 \pm 2{ }^{\circ} \mathrm{C}\right.$ and $60 \pm 10 \%$ 
humidity) and maintained on a purified basal diet or a high-cholesterol diet. The basal diet contained $20 \%$ casein. $63.2 \%$ sucrose. $10 \%$ corn oil. $2 \%$ agar. $0.8 \%$ vitamin mixture and $4 \%$ salt mixture. The high-cholesterol diet consisted of the basal diet with $1.5 \%$ cholesterol and $0.5 \%$ cholic acid in place of an equal amount of sucrose.

Experimental design: Animals were divided into four groups: Group I (Basal) $(n=6)$. Group II (Chol) $(n=6)$. Group III (Basal+Adj) $(n=9)$, and Group IV (Chol+ Adj) $(n=9)$. Groups I and III were fed the basal diet. Groups II and IV were fed the high-cholesterol diet. After feeding of each diet for 10 days, each rat in Groups III and IV received adjuvant, a fine suspension of killed Mvcobacterium butyricum (Difco Laboratories). $0.6 \%(\mathrm{w} / \mathrm{v})$, in liquid paraffin. The diet was given by pair-feeding, and water was freely available. The rats were weighed weekly during the experiment.

Induction of arthritis: Adjuvant arthritis was induced by a single s.c. injection of $0.1 \mathrm{ml}$ adjuvant into the base of the rat tail

Evaluation of arthritis: Both hind paw volumes were measured by water displacement to the lateral malleolus (ankle) just before adjuvant injection and then, to follow the development of the inflammatory lesions, at intervals of 3-4 days. The percentage changes in paw volume were calculated using the following equation:

Percentage change $=(Y-X) / X \times 100$

where $X=$ the mean value of both hind paw volumes before injection and $Y=$ the mean value of both hind paw volumes after injection.

The arthritis score was also determined by an index described previously (10), with the four paws and tail being graded from 0 to 4 based on periartricular erythema, swelling and ankylosis. The maximum possible score was 20.

Sampling from tissues: Throughout the experimental period, blood was drawn from the tail vein at biweekly intervals for cholesterol determination after the animals had fasted overnight. The serum was separated by centrifugation (1500 $\times g$ for 10 $\mathrm{min}$ ) and stored at $-20^{\circ} \mathrm{C}$ until analysis. At the end of the experimental period ( 8 weeks). the rats, after overnight fasting. were sacrificed under ether anesthesia by bleeding from a cannula inserted into the abdominal aorta close to the iliac bifurcation. The liver and aorta, from the arch to the iliac bifurcation. were then excised. Immediately after the serum had been separated, high-density lipoprotein (HDL) fractions were separated from it by the heparin-manganese precipitation procedure (11). The aorta was freezedried to a constant weight and cut into small segments, and the lipids were subsequently extracted at $50^{\circ} \mathrm{C}$ for $20 \mathrm{~min}$ with chloroformmethanol $(2: 1, v / v)$. The delipidated tissues were transferred to hydrolysis tubes, mixed with $6 \mathrm{~N} \mathrm{HCl}$, flushed with nitrogen, sealed and hydrolyzed for $24 \mathrm{hr}$ at $105^{\circ} \mathrm{C}$. Hydrolysates were evaporated under vacuum and used to determine the contents of calcium, phosphorus, hydroxyproline and proline. The ash from the $\mathrm{HCl}$ hydrolysate treated with concentrated nitric acid was used to determine the amount of sulfate. The liver lipids were extracted by the method of Folch et al. (12).

Analytical methods: Total and free cholesterol levels in serum, aorta and liver were fluoroenzymatically estimated as described previously (13). Triglycerides were determined by the acetylacetone method of Fletcher (14). Lipid peroxides were fluorometrically determined by the thiobarbituric acid (TBS) method of Yagi (15). Hydroxyproline and proline were determined by the method of Rojkind and González (16). The amount of calcium in the aorta was determined with an atomic absorption spectrophotometer (Model 180-60. Hitachi). Phosphorus was determined by the method of McClare (17). Sulfate was determined by the method of Antonopoulos (18) except that 2,7-diaminofluorene (Nakarai Chemicals) was substituted for benzidine.

Statistics: The results obtained were expressed as the mean \pm S.E. Student's $t$-test for paired observations was used to test for significance.

\section{Results}

Body weight: Changes in the body weight are shown in Fig. 1. Despite pair-feeding, the animals in Groups I and II without adjuvant 


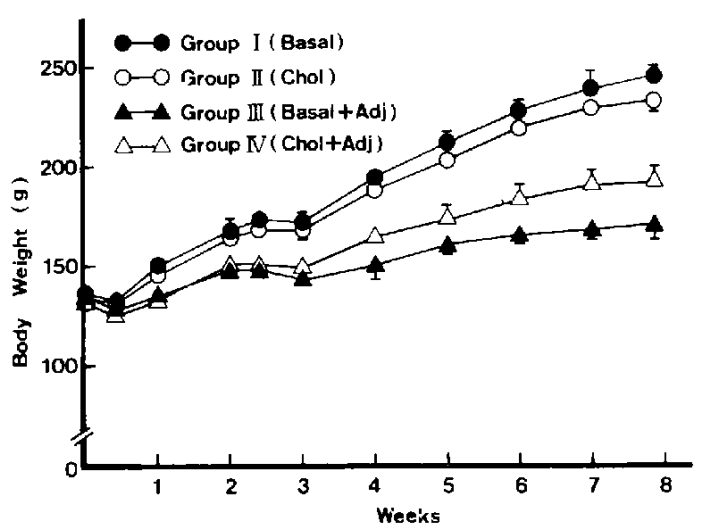

Fig. 1. Growth rate of various rat groups. Number of animals: 6/adjuvant non-treated group (Groups 1. II) and g/adjuvant treated group (Groups III, IV). Each point represents the meantS.E. Group composition is detailed in Materials and Methods.

treatment gained weight relatively steadily throughout the experimental period. However, the growth rates of the groups that received adjuvant (Groups III and IV) were significantly inhibited compared to the control group (Group I) after 1 week. Group IV showed a tendericy of increase in body weight compared to Group III, although the values were not statistically significant. Adjuvant-treated animals left a considerable amount of uneaten food, especially the few days after adjuvant injection and around three weeks after the injection, during the developmental stage of arthritis.

Time course of serum cholesterol: As shown in Fig. 2, at the starting stage of the experiment, the serum total cholesterol levels in Groups II and IV. which were given the high-cholesterol diet for 10 days before the adjuvant injection, were already approx. three times higher than those of the groups given the basal diet (Groups I and III). Thereafter, continuous high levels were retained in these groups throughout the experimental period. The cholesterol levels in Group IV tended to be higher than those in Group II.

Time course of the rat adjuvant response: Figures 3 and 4 show the time curves for the development of inflammation as reflected by the paw volume and arthritis score, respectively. All of the rats (Groups III and IV) that

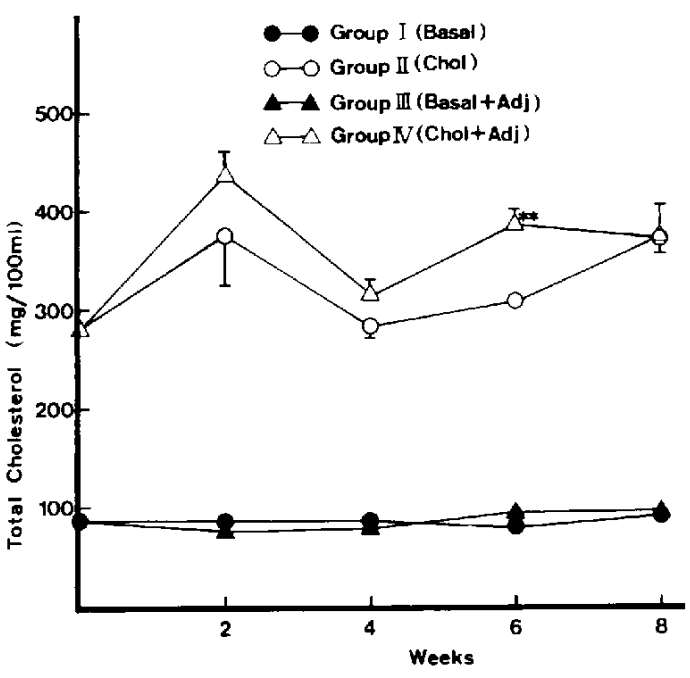

Fig. 2. Time course of serum cholesterol levels. Rats were fed the basal or high-cholesterol diet from 10 days before the start of the experiment (adjuvant injection) to the 8th week. Number of animals: 6/adjuvant non-treated group (Groups I. II) and 9/adjuvant treated group (Groups III. IV). Each point represents the mean \pm S.E. ${ }^{*} P<0.01$, as compared with Group 11. Group composition is detailed in Materials and Methods.

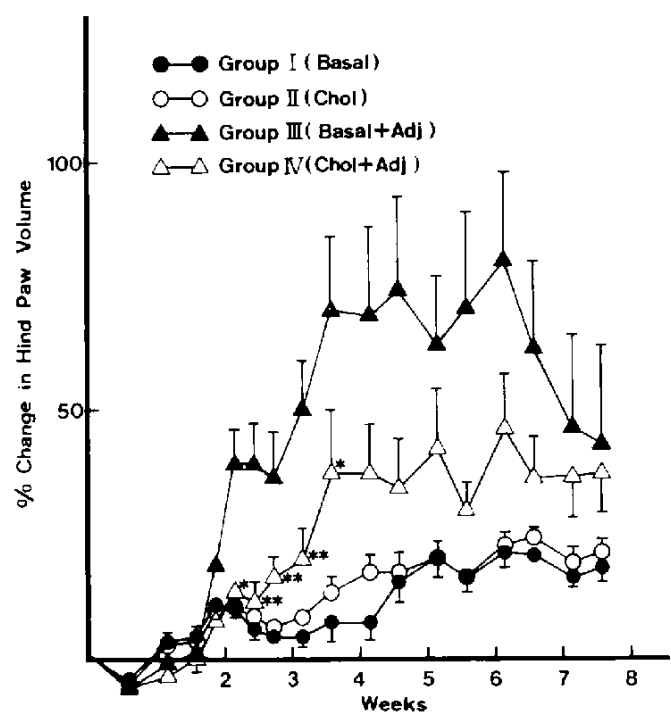

Fig. 3. Time course of percentage change in both hind paw volumes after adjuvant injection. Each point represents the mean \pm S.E. ${ }^{*} \mathrm{P}<0.05,{ }^{* *} \mathrm{P}<0.01$. as compared with Group III. For other references, see the legend to Fig. 2. 
received a single adjuvant injection developed arthritis. Animals in Group III developed severe polyarthritis which became apparent at week 2. peaked at week 4 and persisted until week 6 after adjuvant injection. On the other hand, in the cholesterol-fed group (Group IV), the onset of arthritis was fairly delayed and the inflammatory response was remarkably suppressed, especially in the early stage of arthritis development.

Lipid levels in serum: Serum lipid levels at

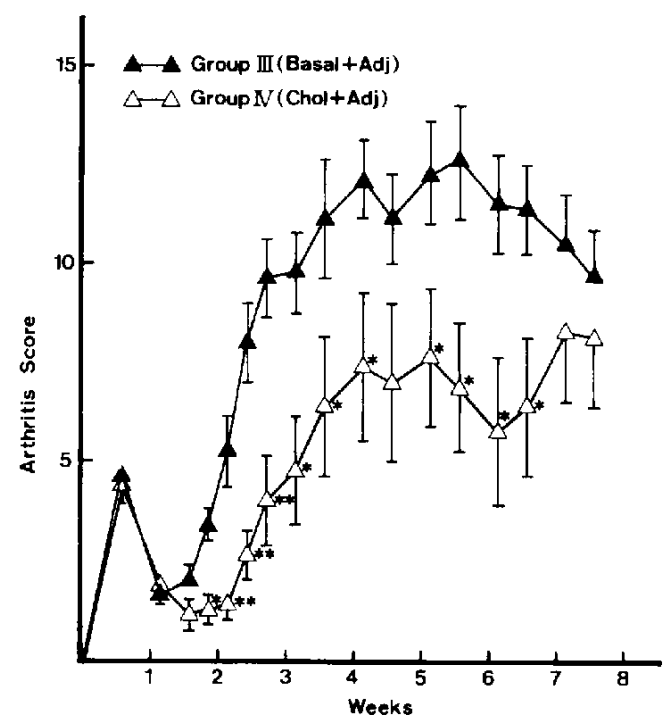

Fig. 4. Time course of inflammatory change in the four paws and tail after adjuvant injection. Each point represents the mean $\pm S . E .{ }^{*} P<0.05,{ }^{*} \mathrm{P}<0.01$, as compared with Group III. For other references. see the legend to Fig. 2. the end of the experiment are given in Table 1. Cholesterol feeding resulted in marked increases in both free and esterified cholesterol levels, but adjuvant treatment had no influence on them. HDL-cholesterol levels were remarkably low in rats fed the highcholesterol diet compared to the respective basal diet control. Adjuvant treatment caused a slight but significant increase in the HDLcholesterol levels in rats fed the basal diet, but had no effect in rats fed the high-cholesterol diet. Serum triglyceride levels significantly decreased after either cholesterol feeding or adjuvant treatment $(P<0.01$. Group I vs. II and I vs. III, respectively). Lipid peroxide levels were significantly decreased by cholesterol feeding ( $P<0.01$. Group I vs. II and III vs. IV. respectively). Adjuvant treatment caused a slight decrease in lipid peroxide level, but potentiated the lowering effect by cholesterol feeding (statistically significant difference, $P<0.01$, Group II vs. IV)

Lipid levels in aorta and liver: Table 2 shows the marked increase (27\%) in the aortic total cholesterol level in Group IV animals, which had received both the highcholesterol diet and adjuvant treatment, compared to the control group: no significant difference was found among Groups I, II and III. Both aortic free and esterified cholesterol levels were also markedly increased by the combination of cholesterol feeding and adjuvant treatment in parallel with the total cholesterol level. Triglyceride levels of the aorta were significantly decreased by adjuvant

Table 1. Serum lipid levels of various rat groups

\begin{tabular}{|c|c|c|c|c|}
\hline & \multicolumn{3}{|c|}{ Group } & \\
\hline & ( Basal) & II (Chol) & III (Basal+Adj) & IV (Chol+Adj) \\
\hline$n$ & 6 & 6 & 9 & 9 \\
\hline Total cholesterol (mg/100 ml) & $92 \pm 4$ & $371 \pm 17^{* *}$ & $96 \pm 2$ & $370 \pm 37^{* *}$ \\
\hline Free cholesterol $(\mathrm{mg} / 100 \mathrm{ml})$ & $34 \pm 1$ & $64 \pm 2^{* *}$ & $36 \pm 1$ & $73 \pm 6^{* *}$ \\
\hline Cholesterol ester (mg/100 $\mathrm{ml}$ ) & $58 \pm 4$ & $307 \pm 15^{+*}$ & $61 \pm 2$ & $297 \pm 31^{* *}$ \\
\hline $\mathrm{HDL}$-cholesterol $(\mathrm{mg} / 100 \mathrm{ml})$ & $39.8 \pm 2.0$ & $10.8 \pm 1.1 * *$ & $45.6 \pm 1.4^{*}$ & $8.0 \pm 1.5^{* *}$ \\
\hline Triglycerides (mg/100 $\mathrm{mi}$ ) & $199 \pm 18$ & $46=10^{+*}$ & $90 \pm 17^{* *}$ & $37 \pm 6^{* *}$ \\
\hline Lipid peroxide $(\mathrm{nmol} / \mathrm{ml})$ & $10.1 \pm 1.0$ & $5.1 \pm 0.3^{* *}$ & $8.2 \pm 0.5$ & $3.4 \pm 0.3^{* * * * * * t ;}$ \\
\hline
\end{tabular}

Rats were killed 8 weeks after adjuvant injection. Group composition is detailed in Materials and Methods. $n$ : Number of animals. Each value represents the mean \pm S.E. ${ }^{*} P<0.05,{ }^{* *} P<0.01$, as compared with Group 1. \#\#: $P<0.01$, as compared with Group II. Lipid peroxide is expressed as the amount of thiobarbituric acid reacting substance. 
Table 2. Aortic and liver lipid levels of various rat groups

\begin{tabular}{|c|c|c|c|c|c|}
\hline \multirow{3}{*}{\multicolumn{2}{|c|}{ n }} & \multicolumn{3}{|c|}{ Group } & \multirow[b]{2}{*}{ IV (Chol+Adj) } \\
\hline & & I (Basal) & II (Chol) & III (Basal + Adj) & \\
\hline & & 6 & 6 & 9 & 9 \\
\hline \multicolumn{6}{|c|}{ Aorta (mg/g dry weight) } \\
\hline Total cholesterol & & $5.42 \pm 0.06$ & $5.67 \pm 0.16$ & $5.34 \pm 0.09$ & $6.90 \pm 0.30 * * m *$ \\
\hline Free cholesterol & & $5.19 \pm 0.06$ & $5.34 \pm 0.20$ & $5.05 \pm 0.05$ & $6.14 \pm 0.14^{* * * * *}$ \\
\hline Cholesterol ester & & $0.23 \pm 0.003$ & $0.32 \pm 0.04$ & $0.29 \pm 0.03$ & $0.76 \pm 0.18^{* * \#}$ \\
\hline Triglycerides & & $22.1 \pm 3.3$ & $35.1 \pm 5.0$ & $8.4 \pm 0.8^{* *}$ & $8.6 \pm 1.2^{* * * \#}$ \\
\hline \multicolumn{6}{|c|}{ Liver ( $\mathrm{mg} / \mathrm{g}$ wet weight) } \\
\hline Total cholesterol & & $2.2 \pm 0.1$ & $87.9 \pm 9.8^{* *}$ & $3.0 \pm 0.2^{*}$ & $99.4 \pm 5.3^{* *}$ \\
\hline Free cholesterol & & $1.9 \pm 0.1$ & $3.0 \pm 0.1^{*}$ & $2.3 \pm 0.2$ & $3.7 \pm 0.3^{* *}$ \\
\hline Cholesterol ester & & $0.3 \pm 0.03$ & $84.8 \pm 9.7^{* *}$ & $0.7 \pm 0.1$ & $95.8 \pm 5.1^{* *}$ \\
\hline Triglycerides & & $11.0 \pm 1.6$ & $48.6 \pm 2.9^{* *}$ & $12.5 \pm 1.5$ & $35.2 \pm 3.4^{* * * *}$ \\
\hline
\end{tabular}

Rats were killed 8 weeks after adjuvantinjection. Group composition is detailed in Materials and Methods. $n$ : Number of animals. Each value represents the mean $+S . E$. ${ }^{*} P<0.05,{ }^{*} P<0.01$, as compared with Group 1. "P<0.05, " $\mathrm{P}<0.01$, as compared with Group II.

Table 3. Aortic connective tissue component and mineral levels of various rat groups

\begin{tabular}{|c|c|c|c|c|c|}
\hline & & & $\mathrm{Gr}$ & & \\
\hline & & | (Basal) & II (Chol) & III (Basal+Adj) & IV (Chol + Adj) \\
\hline & $n$ & 6 & 6 & 9 & 9 \\
\hline & & & & $\mathrm{mg} / \mathrm{g}$ dry weight & \\
\hline Hydroxyproline & & $56.8 \pm 0.5$ & $57.5 \pm 1.0$ & $55.2 \pm 1.4$ & $54.5 \pm 1.4$ \\
\hline Proline & & $89.6 \pm 2.3$ & $93.2 \pm 1.9$ & $92.6 \pm 1.0$ & $93.0 \pm 1.4$ \\
\hline Sulfate & & $11.1 \pm 0.3$ & $11.1 \pm 0.3$ & $11.6 \pm 0.3$ & $11.5 \pm 0.3$ \\
\hline Calcium & & $0.244 \pm 0.035$ & $0.288 \pm 0.017$ & $0.226 \pm 0.032$ & $0.236 \pm 0.012$ \\
\hline Phosphorus & & $0.807 \pm 0.023$ & $0.833 \pm 0.032$ & $0.775 \pm 0.024$ & $0.802 \pm 0.017$ \\
\hline
\end{tabular}

Rats were killed 8 weeks after adjuvant injection. Group composition is detailed in Materials and Methods. n: Number of animals. Each value represents the mean $\pm S . E$.

treatment, but not by cholesterol feeding.

Both liver cholesterol and triglyceride levels were markedly increased by feeding with cholesterol. Adjuvant treatment tended to increase the liver cholesterol levels.

Connective tissue component levels in the aorta: As shown in Table 3, neither the high-cholesterol diet nor the treatment with adjuvant produced any significant changes in the levels of hydroxyproline, proline, sulfate. calcium and phosphorus in the aorta.

\section{Discussion}

The results of this experiment demonstrate that in the rat, adjuvant arthritis and cholesterol feeding markedly increase the choles terol level in the aorta, whereas either of these treatments alone had hardly any effect. This indicates the synergy of chronic inflammatory responses and hypercholesterolemia for lipid deposition on the arterial wall. These results biochemically confirm the observations of Virág et al. (8) that increased amounts of lipid deposits can be histologically demonstrated by adjuvant arthritis combined with a cholesterol diet in the abdominal aorta and various arteries of rats; the thoracic aorta was not examined.

Virág et al. (8) also suggested that the major lipids which accumulate in the aorta of animals treated with adjuvant and a cholesterol diet are cholesterol and triglycerides, based on histological examination. In this study. the accumulated lipids were predominantly cholesterol, particularly cholesterol ester, while the triglyceride levels 
in the aorta were actually rather reduced. We do not yet know why the aortic triglyceride level was low or whether it resulted from the decreased serum triglyceride level. Lipids of the atherosclerotic plaque are known to exist as complexes of lipoprotein, acid mucopolysaccharides, and calcium (19). Virág et al. (8) have further emphasized definite increases of mucopolysaccharides together with the lipid deposits in the aorta. However. in this study. no increase in mucopolysaccharides was observed judging from the levels of sulfate, their marker, in the total aorta. The amounts of the other connective tissue components and minerals also remained unchanged. These findings may indicate that the earlier atherosclerotic lesions without appreciable intimal or medial change were successfully produced in this study.

Atherosclerosis is generally believed to be a disease of multiple interacting causative factors. Steinberg (20) has proposed a pathogenetic mechanism combining the lipid hypothesis with the endothelial injury hypothesis. Hypercholesterolemia, or low density lipoprotein (LDL) elevation, may itself damage the arterial endothelium, and increase the rate of penetration of lipoproteins into the artery wall. In rabbits, atherosclerosis can be established by cholesterol feeding alone. However, the rat is quite resistant and cholesteral feeding alone does not produce atherosclerotic lesions. In previous studies $(21,22)$ as well as in the present one, no significant accumulation of cholesterol in the aorta was observed in rats, despite the fact that sufficient hypercholesterolemia was achieved by cholesterol feeding alone. For the rat, vascular injury in combination with hypercholesterolemia is necessary for lipids to accumulate on the arterial wall $(3,8,22$, 23). The present experiments also demonstrated that adjuvant treatment in addition to cholesterol feeding is essential for causing cholesterol accumulation in the aorta. The development of adjuvant arthritis in the rat is thought to be a cell-mediated delayed type hypersensitivity reaction to mycobacterial fragments $(24,25)$. Also, immunological injury to the vessel wall promotes atherosclerosis, as has been shown by the induction of serum sickness in the rat (3). Therefore. adjuvant treatment probably exerts an injuring action on vessels as well as joints.

Interestingly, we found that cholesterol feeding inhibited the induction of adjuvant arthritis, although cholesterol has no antiinflammatory effect. The role of cholesterol in adjuvant arthritis has not been studied, but we observed that cholesterol feeding could lower the serum lipid peroxide level. Similar results have been obtained in a previous study (21). The relation of lipid peroxidation to adjuvant arthritis (26) and rheumatoid arthritis (27) has been reported. Therefore, cholesterol may act by lowering the serum lipid peroxide level. Cholesterol is essential to the structure of all cell membranes and is a precursor of all steroid hormones. Obviously. there are many mechanisms by which cholesterol may interact with the induction process of adjuvant arthritis. Further studies are needed to clarify them.

Our study with this animal model of atherosclerosis provided biochemical evidence for the association between chronic inflammation, resulting from immunological reactions, and lipid accumulation on the arterial wall in hypercholesterolemic rats.

\section{References}

1 Ross, R. and Glomset, J.A.: The pathogenesis of atherosclerosis. N. Engl. J. Med. 295, 369-377. 420-425 (1976)

2 Reidy, M.A. and Schwartz, S.M.: Endothelial injury and regeneration. IV. Endotoxin: A nondenuding injury to aortic endothelium. Lab. Invest. 48, 25-34 (1983)

3 Horsch, A.K., Kuhlmann, W.D., Bleyl, U. and Salomon, J.C.: Early atherosclerotic lesions in rat aurta. Effect of cholesterol feeding and immunization. Res. Exp. Med. 173, 251-259 (1978)

4 Minick, C.R.: Immunologic arterial injury and atherogenesis. In Atherosclerosis, Edited by Gotto, A.M., Jr., Smith, L.C. and Allen, B., Vol. 5, p. 330-333. Springer-Verlag. New York. Heidelberg and Berlin (1980)

5 Meller, J., Conde, C.A., Deppisch, L.M., Donoso, E. and Dack, S.: Myocardial infarction due to coronary atherosclerosis in three young adults with systemic lupus erythematosus. Am. J. Cardiol. 35, 309-314 (1975)

6 Vikhert, A.M. and Zhdanov, V.S.: Atherosclerosis and rheumatic heart disease. In the Effects of 
Various Diseases on the Development of Atherosclerosis, Edited by Muiler, J.E., p. 89-103. Pergamon Press, New York (1981)

7 Accinni, L. and Dixon, F.J.: Degenerative vascular disease and myocardial infarction in mice with lupus-like syndrome. Am. J. Pathol. 96, 477-492 (1979)

8 Virág, S., Vértesi, C. and Welner, I.: Combined vessel injuring action of adjuvant arthritis and cholesterol feeding. 1. A new method for producing experimental atherosclerosis in rats. Ther. Hung. 24, 137-141 (1976)

9 Virág, S., Vértesi, C. and Welner, I.: Combined vessel injuring action of adjuvant arthritis and cholesterol feeding. II. The influence of different types of drugs on the new model of experimental atherosclerosis. Ther. Hung. 24, 142-144 (1976)

10 Koga, T. and Pearson, C.M.: Immunogenicity and arthritogenicity in the rat of an antigen from Mycobacterium tuberculosis wax D. J. Immunol. 111, 599-608 (1973)

11 Warnick, G.R. and Albers, J.J.: A comprehensive evaluation of the heparin-manganese precipitation procedure for estimating high density lipoprotein cholesterol. J. Lipid Res. 19, 65-76 (1978)

12 Folch, J., Lees, M. and Stanley, G.H.S.: A simple method for the isolation and purification of total lipids from animal tissues. J. Biol. Chem. 226, 497-509 (1957)

13 Kunitomo, M., Yamaguchi, Y., Matsushima, K. and Bandô, $Y$.: Cholesterol metabolism in serum and aorta of inbred mice fed a high-cholesterol diet. Japan. J. Pharmacol. 34, 153-158 (1984)

14 Fletcher, M.J.: A colorimetric method for estimating serum triglycerides. Clin. Chim. Acta 22, 393-397 (1968)

15 Yagi, K.: A simple fiuorometric assay for lipoperoxide in blood plasma. Biochem. Med. 15 , 212-216 (1976)

16 Rojkind, M. and González, E.: An improved method for determining specific radioactivities of proline- ${ }^{14} \mathrm{C}$ and hydroxyproline- ${ }^{14} \mathrm{C}$ in collagen and in noncollagenous proteins. Anal. Biochem. 57, 1-7 (1974)

17 McClare, C.W.F.: An accurate and convenient organic phosphorus assay. Anal. Biochem. 39.
$527-530(1971)$

18 Antonopoulos, C.A.: A modification for the determination of sulfate in mucopolysaccharides by the benzidine method. Acta Chem. Scand. 16, 1521-1522 (1962)

19 Hollander, W.: Unified concept on the role of acid mucopolysaccharides and connective tissue proteins in the accumulation of lipids and lipoproteins and calcium in atherosclerotic plaques. Exp. Mol. Pathol. 25, 106-120 (1976)

20 Steinberg, D.: Metabolism of lipoproteins at the cellular level in relation to atherosclerosis. In Metabolic Aspect of Cardiovascular Disease. Edited by Miller. N.E. and Lewis, B., Vol. 1, p. 31-48. Elsevier. North-Holland Biomedical Press, Amsterdam, New York and Oxford (1981)

21 Kunitomo, M., Yamaguchi, Y., Matsushima, K., Futagawa, $Y$. and Bandô, Y.: Hyperlipidemic effects of adriamycin in rats. Japan. J. Pharmacol. 39, 323-329 (1985)

22 Kunitomo, M., Kinoshita, $K$. and Bandô, $Y$ : Experimental atherosclerosis in rats fed a vitamin D. cholesteral-rich diet. J. Pharmacobiodyn. 4, 718-723 (1981)

23 Koletsky, S. and Snajdar, R.M.: Atherosclerosis following balloon catheter injury to the carotid artery and the aorta of hypertensive rats with normolipidemia or hyperlipidemia. Am. J. Pathol. 103, 105-115 (1981)

24 Kayashima, K., Koga, T. and Onoue, K.: Role of T lymphocytes in adjuvant arthritis. II. Different subpopulations of $T$ Iymphocytes functioning in the development of the disease. J. Immunol. 120, 1127-1131 (1978)

25 Gans, K.R., Heyner, S. and Orzechowski, R.F.: Application of a radiometric ear assay for studies of adjuvant arthritis in rats. Arthritis Rheum. 23, $633-640$ (1980)

26 Robak, J.: Adjuvant-induced and carrageenininduced inflammation and lipid peroxidation in rat liver, spleen and lungs. Biochem. Pharmacol. 27, 531-533 (1978)

27 Yoshikawa, T., Yokoe, N., Takemura, S., Kato, H., Hosokawa, K. and Kondo, Mi: Studies on the pathogenesis of rheumatoid arthritis. 1. Lipid peroxide and lysosomal enzymes in rheumatoid joints. Japan. J. Med. 18, 199-204 (1979) 